\title{
Performances Comparison for a Rotating Shaft Suspended by 4-Axis Radial Active Magnetic Bearings via $\mu$-Synthesis, Loop-Shaping Design, and $\operatorname{Sub}(H)_{\infty}$ with Uncertainties
}

\author{
G. Barbaraci and G. Virzi' Mariotti \\ Dipartimento di Ingegneria Chimica, Gestionale, Informatica e Meccanica, Universita' di Palermo, Viale delle Scienze, \\ 90128 Palermo, Italy \\ Correspondence should be addressed to G. Virzi’ Mariotti, gabriele.virzimariotti@unipa.it
}

Received 3 March 2011; Revised 8 April 2011; Accepted 9 May 2011

Academic Editor: Jing-song Hong

Copyright ( $) 2011$ G. Barbaraci and G. Virzi’ Mariotti. This is an open access article distributed under the Creative Commons Attribution License, which permits unrestricted use, distribution, and reproduction in any medium, provided the original work is properly cited.

\begin{abstract}
The control systems applied on active magnetic bearing are several. A perfect levitation is characterized by maintaining the operating point condition that is characterized by the center of stator coincident with the geometric center of shaft. The first controller implemented for this purpose is PID controller that is characterized by an algorithm that leads the amplifier to produce control current until the operating point condition is not reached, this is obtained by an integration operator. The effect of an integrator is essential but not necessary for a centered levitation for example in the robust control characterized by a dynamic model depended on plant of system so that it depends on angular speed as LQR controller does. In LQR there is not integrator so there is not a perfectly centered section of shaft with center of stator. On contrary PID controller does not depend on angular speed and it can be easily implemented according some simple rules. Predictive control is another interesting controller characterized by a multiple controller operating in different condition in order to get the minimum of cost function, but also in this case the angular speed is introduce for the same reason discussed before.
\end{abstract}

\section{Introduction}

Active Magnetic Bearings (AMBs) use electromagnets to attract the ferromagnetic cape winding the rotor which is free to rotate with no physical contact with the bearing. This operation, called active magnetic levitation, is unstable unless of a certain control's algorithm performed respecting the imposed constraining, [1]. In order to achieve a stable levitation, an active feedback control of the current in the magnetic coils is necessary. As it might be expected, a variety of control schemes are used and a variety of studies have been done for AMBs control. The dynamic system, however, depends above all on the rotor's angular speed, as the case for any rotor dynamic system because of the gyroscopic effect [2]. The gyroscopic effect leads the system into instability phenomena which must be considered to achieve stable levitation. The rotor motion is characterized by translation along $x-y$ directions and rotation of rotor around those axes
$[1,3,4]$. Moreover, the transformation coordinates allow relating the displacements captured by the sensors and the displacements of the section located on the middle plane of the bearings location. Without a suitable control system, no magnetic levitation is possible [1]. During last twenty years, a fast number of control systems were applied on magnetic levitation in order to provide enough acknowledge about the capability of rotor to maintain the contactless between the rotor and stator. Obviously while there are many control systems which are not able to maintain the operating point position of the controlled sections without a further algorithm such as the integrator of a PID controller [5], some other control systems need the entire state vector to create the feedback such as the optimal control [3] characterized by a matrix whose number of column is equal to the dimension of state vector. The cutting edge of control systems is represented by $\mu$-synthesis, loop-shaping design procedure, and $\mathrm{H}$-infinity robust control, the latter with its variant 


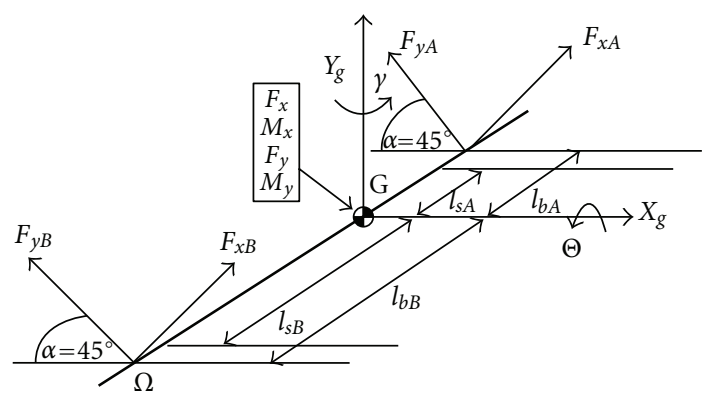

FIGURE 1: Schematic view of 4-axis rotating shaft supported by two radial active magnetic bearings with sensors.

$\operatorname{sub}(H)_{\infty}$. The reason is not only the recovering of operating point position without integrator but also the possibility to delete the use of some sensors to capture further components belonging to the state vector, a problem that is usually solved by the introduction of an observer. There is something more convenient than the previous simplification which is more related to the performances of the machine in using robust control. The advantages derived by using robust control are its possibility in case of presence of dynamic perturbation such as, no modeled dynamic, neglected nonlinearities, effects of reduced-order models, system-parameter variation due to environmental changes, hysteresis, and torn and worn factors. Moreover, it is used also in case there is a presence of sensor and actuator noise. An application of $\mu$-synthesis controller was for the Army's weapon system. The test fixture is patterned after the Apache helicopter's $30 \mathrm{~mm}$ gun and has tunable nonlinearities which may be representative not only of the nonlinearities of the gun, but also to other mechanical systems as well. The models of the test fixture which were available at the time of the work are also described. The goal of pointing the gun is to reduce dispersions of fired gun rounds on targets. The resulting $\mu$-synthesis design, when connected with a nonlinear simulation, exhibited limitcycle behavior of unacceptable amplitude [6]. Due to high surface speed and active control capabilities, active magnetic bearings hold great promise for high speed machining spindles. The control problem posed by this application is examined, and the development of an advanced prototype is reviewed. A $\mu$-synthesis framework is proposed for this problem, and it is shown that the minimization of the susceptibility to machining chatter may be easily put into this framework. In addition to handling uncertainties in sensor and actuator components, this formulation may also include an uncertainty representing the range of cutting tools for the spindle, [7].

The proposed control algorithms are developed using $\mu$ analysis to obtain robust stability and robust performance in simulation investigation. In simulations work, three different active vibration control algorithms are used. A similar approach was applied in [8] where a comparison between three different controllers was performed in order to analyze the differences on the dynamic behavior. Many other applications of robust control which were performed through loop-shaping design procedure such as in [9] where an $H_{\infty}$ controller was performed by evolution optimization to control a robot arm.

The loop-shaping method is commonly used also to obtain tradeoffs of robust stability and robust performance. This technique is a particular optimization problem to guarantee closed loop stability at all frequencies [10].

The particular configuration shown in this work considers a rotor with four degrees of freedom with eight poles for each active magnetic bearing, having a slope of $45^{\circ}$ with regard to horizontal direction so that the force's resultant supports the rotor along the $x$ and $y$ directions crossing the center of mass and rotation around it as Figure 1 shows.

The system is subjected to a state of uncertainty about its mass, cross, and polar moment of inertia dictated by the parameters $\delta_{m}, \delta_{I_{P}}$, and $\delta_{I_{T}}$ in the ranges, respectively, $P_{m}, P_{I_{T}}$, and $\mathrm{P}_{I_{P}}$. The equation of motion is referred to the center of gravity, and it has the following expression:

$$
\mathbf{M} \frac{d^{2} \mathbf{q}_{g}(t)}{d t^{2}}+\Omega \cdot \mathbf{G} \frac{d \mathbf{q}_{g}(t)}{d t}=\mathbf{B}_{\Theta \mathrm{mag}} \mathbf{B}_{\mathrm{mag}} \mathbf{f}\left(\mathbf{i}_{c}(t), \mathbf{q}_{b}(t)\right)
$$

where

$$
\begin{aligned}
& \mathbf{M}=\left[\begin{array}{cccc}
m & 0 & 0 & 0 \\
0 & I_{T} & 0 & 0 \\
0 & 0 & m & 0 \\
0 & 0 & 0 & I_{T}
\end{array}\right], \quad \mathbf{G}=\left[\begin{array}{cccc}
0 & 0 & 0 & 0 \\
0 & 0 & 0 & -I_{P} \\
0 & 0 & 0 & 0 \\
0 & I_{P} & 0 & 0
\end{array}\right], \\
& \mathbf{B}_{\Theta \operatorname{mag}}=\left[\begin{array}{cccc}
\cos (\alpha) & 0 & -\sin (\alpha) & 0 \\
0 & \cos (\alpha) & 0 & \sin (\alpha) \\
\sin (\alpha) & 0 & \cos (\alpha) & 0 \\
0 & -\sin (\alpha) & 0 & \cos (\alpha)
\end{array}\right], \\
& \mathbf{B}_{\mathrm{mag}}=\left[\begin{array}{cccc}
1 & 0 & 1 & 0 \\
-l_{b A} & 0 & l_{b B} & 0 \\
0 & 1 & 0 & 1 \\
0 & l_{b A} & 0 & -l_{b B}
\end{array}\right] \text {, } \\
& \mathbf{f}\left(\mathbf{i}_{c}(t), \mathbf{q}_{b}(t)\right)=\left[\begin{array}{c}
F_{x A}\left(i_{c x A}(t), x_{b A}(t)\right) \\
F_{y A}\left(i_{c y A}(t), y_{b A}(t)\right) \\
F_{x B}\left(i_{c x B}(t), x_{b B}(t)\right) \\
F_{y B}\left(i_{c y B}(t), y_{b B}(t)\right)
\end{array}\right] \text {, } \\
& \mathbf{q}_{g}(t)=\left[\begin{array}{llll}
x_{g}(t) & \gamma(t) & y_{g}(t) & \vartheta(t)
\end{array}\right]^{\mathrm{T}} \\
& m=\bar{m}\left(1+P_{m} \delta_{m}\right)=\bar{m}+\bar{m} \cdot P_{m} \delta_{m}=\bar{m}+\Delta \bar{m}, \\
& I_{T}=\bar{I}_{T}\left(1+P_{I_{T}} \delta_{I_{T}}\right)=\bar{I}_{T}+\bar{I}_{T} \cdot P_{I_{T}} \delta_{I_{T} T}=\bar{I}_{T}+\Delta \bar{I}_{T} \text {, } \\
& I_{P}=\bar{I}_{P}\left(1+P_{I_{P}} \delta_{I_{P}}\right)=\bar{I}_{P}+\bar{I}_{P} \cdot P_{I_{P}} \delta_{I_{P}}=\bar{I}_{P}+\Delta \bar{I}_{P} .
\end{aligned}
$$




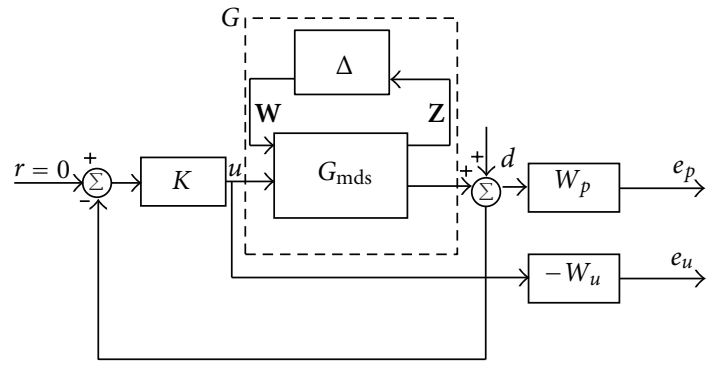

FIGURE 2: Block scheme of plant with the introduction of weighting functions as further outputs.

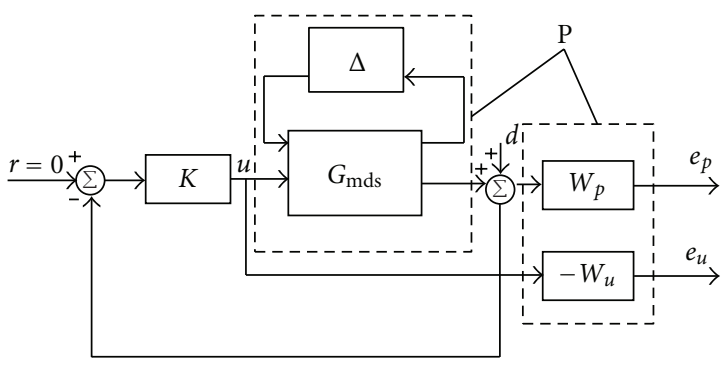

Figure 3: Block scheme of plant showing the new plant.

\section{Mathematical Model}

The last expression leads to structured uncertainties matrix such as

$$
\begin{gathered}
\mathbf{M}=\overline{\mathbf{M}}+\overline{\mathbf{M}} \mathbf{P}_{\mathrm{M}} \Delta_{\mathbf{M}} \in \mathfrak{R}^{4 \times 4}, \\
\mathbf{G}=\overline{\mathbf{G}}+\overline{\mathbf{G}} \mathbf{P}_{\mathrm{G}} \Delta_{\mathrm{G}} \in \mathfrak{R}^{4 \times 4},
\end{gathered}
$$

where

$$
\begin{aligned}
& \overline{\mathbf{M}}=\left[\begin{array}{cccc}
\bar{m} & 0 & 0 & 0 \\
0 & \bar{I}_{T} & 0 & 0 \\
0 & 0 & \bar{m} & 0 \\
0 & 0 & 0 & \bar{I}_{T}
\end{array}\right], \quad \overline{\mathbf{G}}=\left[\begin{array}{cccc}
0 & 0 & 0 & 0 \\
0 & 0 & 0 & -\bar{I}_{P} \\
0 & 0 & 0 & 0 \\
0 & \bar{I}_{P} & 0 & 0
\end{array}\right], \\
& \overline{\mathbf{M}} \mathbf{P}_{M} \Delta_{M}=\overline{\mathbf{M}}\left[\begin{array}{cccc}
P_{m} & 0 & 0 & 0 \\
0 & P_{I_{T}} & 0 & 0 \\
0 & 0 & P_{m} & 0 \\
0 & 0 & 0 & P_{I_{T}}
\end{array}\right]\left[\begin{array}{cccc}
\delta_{m} & 0 & 0 & 0 \\
0 & \delta_{I_{T}} & 0 & 0 \\
0 & 0 & \delta_{m} & 0 \\
0 & 0 & 0 & \delta_{I_{T}}
\end{array}\right], \\
& \overline{\mathbf{G}} \mathbf{P}_{G} \Delta_{G}=\overline{\mathbf{G}}\left[\begin{array}{cccc}
0 & 0 & 0 & 0 \\
0 & P_{I_{P}} & 0 & 0 \\
0 & 0 & 0 & 0 \\
0 & 0 & 0 & P_{I_{P}}
\end{array}\right]\left[\begin{array}{cccc}
0 & 0 & 0 & 0 \\
0 & \delta_{I_{P}} & 0 & 0 \\
0 & 0 & 0 & 0 \\
0 & 0 & 0 & \delta_{I_{P}}
\end{array}\right],
\end{aligned}
$$

respectively, the mass and gyroscopic nominal matrix and structured uncertainties matrices relative to mass and gyroscopic effect.
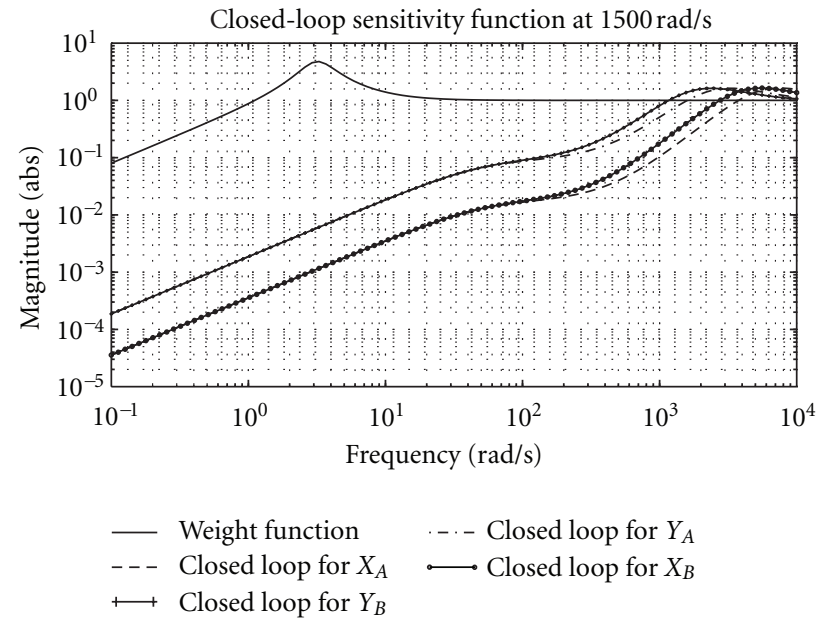

FIgURE 4: Sensitivity function describing how the disturbance affects the displacement with LSDP controller.

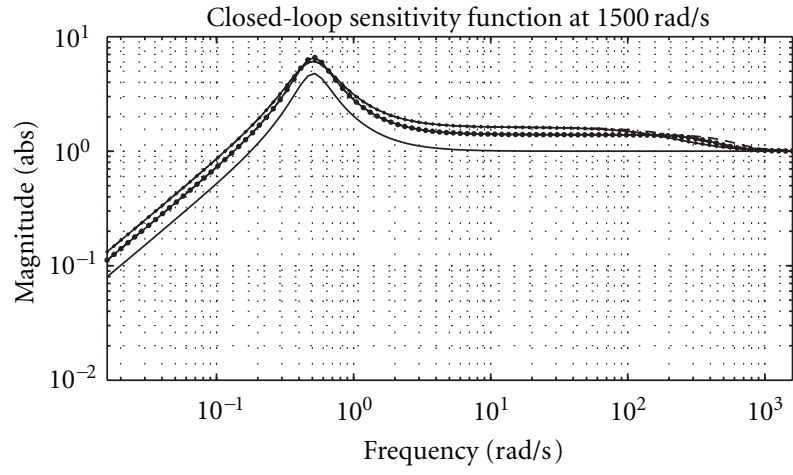

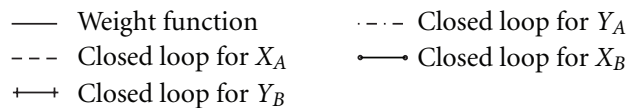

Figure 5: Sensitivity function describing how the disturbance affects the displacement with $\mu$-synthesis controller.

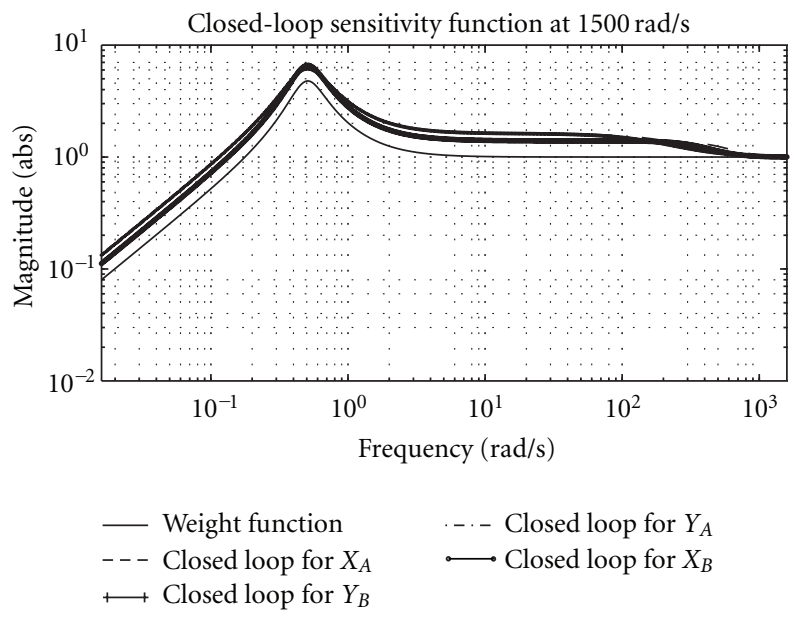

Figure 6: Sensitivity function describing how the disturbance affects the displacement with $\operatorname{Sub}\left(H_{\infty}\right)$ controller. 

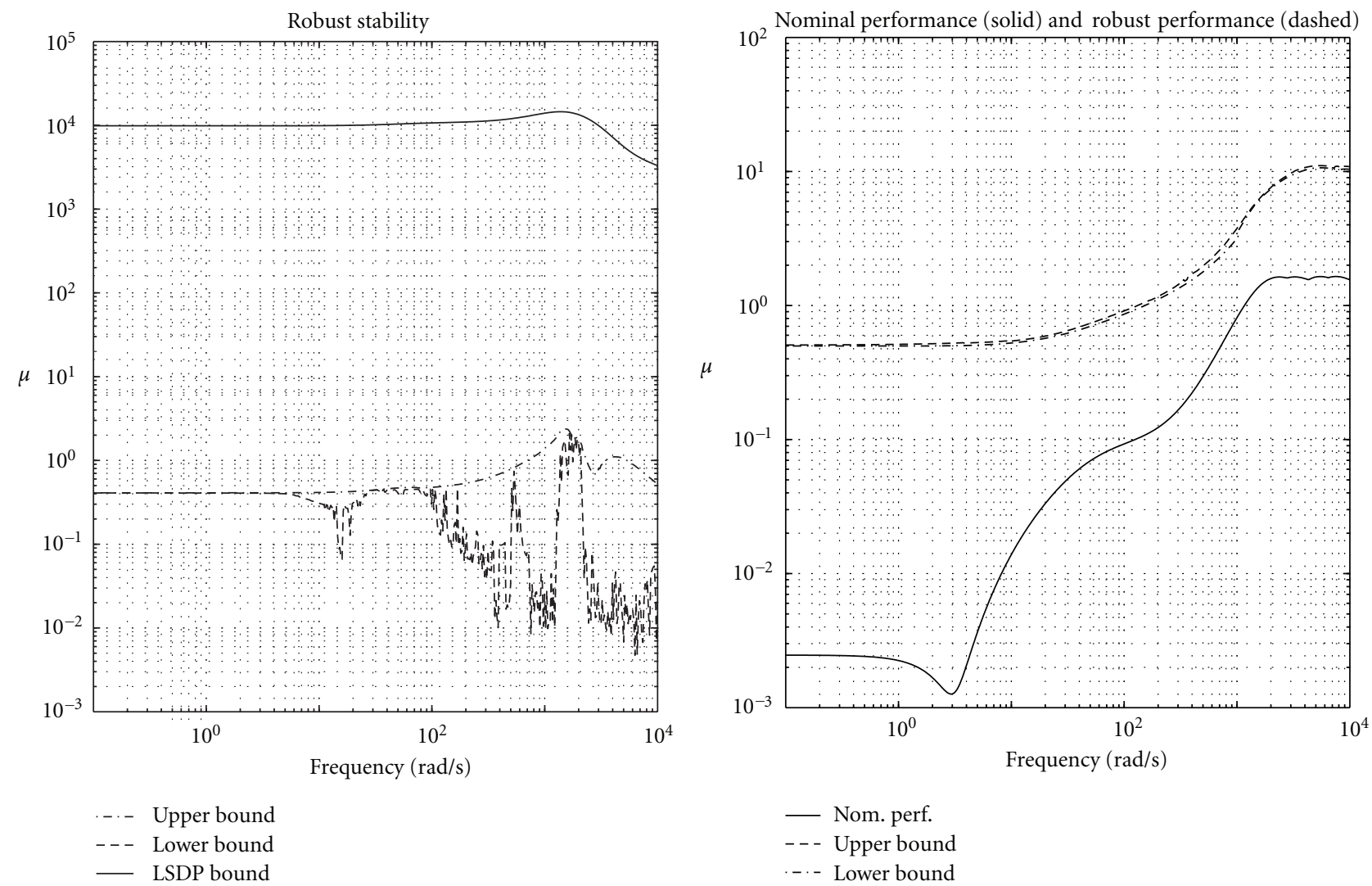

(a)

(b)

FIGURE 7: Robust stability, nominal and robust performance function described by $\mu$ value with LSDP controller.

By introducing a transformation of coordinates (5), which has a great consideration in the relation of the displacements between the section relative to bearing location and the sensors, the system is analyzed according the equation of motion (6):

$$
\begin{gathered}
\mathbf{q}_{b}(t)=\mathbf{B}_{\Theta \text { disp }} \mathbf{B}_{\text {mag }}^{\mathrm{T}} \mathbf{q}_{g}(t), \\
\mathbf{q}_{\text {sensor }}(t)=\left(\mathbf{B}_{\Theta \text { disp }} \mathbf{B}_{\text {sensor }}\right) \mathbf{q}_{g}(t), \\
\mathbf{q}_{\text {sensor }}(t)=\left(\mathbf{B}_{\Theta \text { disp }} \mathbf{B}_{\text {sensor }}\right)\left(\mathbf{B}_{\Theta \text { disp }} \mathbf{B}_{\text {mag }}^{\mathrm{T}}\right)^{-1} \mathbf{q}_{b}(t), \\
\mathbf{f}_{\text {mag_to } \_g}(t)=\mathbf{B}_{\Theta \text { mag }} \mathbf{B}_{\mathrm{mag}} \mathbf{f}\left(\mathbf{i}_{c}(t), \mathbf{q}_{b}(t)\right), \\
\mathbf{M} \frac{d^{2} \mathbf{q}_{g}}{d t^{2}}+\Omega \cdot \mathbf{G} \frac{d \mathbf{q}_{g}}{d t}=\mathbf{B}_{\Theta \mathrm{mag}} \mathbf{B}_{\mathrm{mag}} \mathbf{f}\left(\mathbf{u}(t), \mathbf{q}_{b}(t)\right),
\end{gathered}
$$

where

$$
\mathbf{B}_{\Theta \text { disp }}=\left[\begin{array}{cccc}
\cos (\alpha) & \sin (\alpha) & 0 & 0 \\
-\sin (\alpha) & \cos (\alpha) & 0 & 0 \\
0 & 0 & \cos (\alpha) & \sin (\alpha) \\
0 & 0 & -\sin (\alpha) & \cos (\alpha)
\end{array}\right] \text {, }
$$

$$
\mathbf{B}_{\text {sensor }}=\left[\begin{array}{cccc}
1 & -l_{s A} & 0 & 0 \\
0 & 0 & 1 & l_{s A} \\
1 & l_{s B} & 0 & 0 \\
0 & 0 & 1 & -l_{s B}
\end{array}\right]
$$

$$
\begin{gathered}
\mathbf{q}_{b}(t)=\left[\begin{array}{llll}
x_{b A}(t) & y_{b A}(t) & x_{b B}(t) & y_{b B}(t)
\end{array}\right]^{\mathrm{T}}, \\
\mathbf{q}(t)_{\text {sensor }}=\left[\begin{array}{llll}
x_{s A}(t) & y_{s A}(t) & x_{s B}(t) & y_{s B}(t)
\end{array}\right]^{\mathrm{T}} .
\end{gathered}
$$

The magnetic force (9a)-(9d) produced by an electromagnet of active magnetic bearing is linearized by Taylor series expansion which leads to the expression of the force (9a), [5]:

$$
\begin{array}{r}
f\left(i_{c c(i x, i y)(A, B)}(t), q_{b(x, y)(A, B)}(t)\right) \\
=k\left[\left(\frac{i_{0(A, B)} \mp i_{c(i x, i y)(A, B)}}{g_{0(A, B)} \pm q_{b(x, y)(A, B)}}\right)^{2}\right], \\
\mathbf{f}\left(\mathbf{i}_{c}(t), \mathbf{q}_{b}(t)\right) \approx \mathbf{K}_{S} \mathbf{q}_{b}(t)+\mathbf{K}_{I} \mathbf{i}_{c}(t),
\end{array}
$$




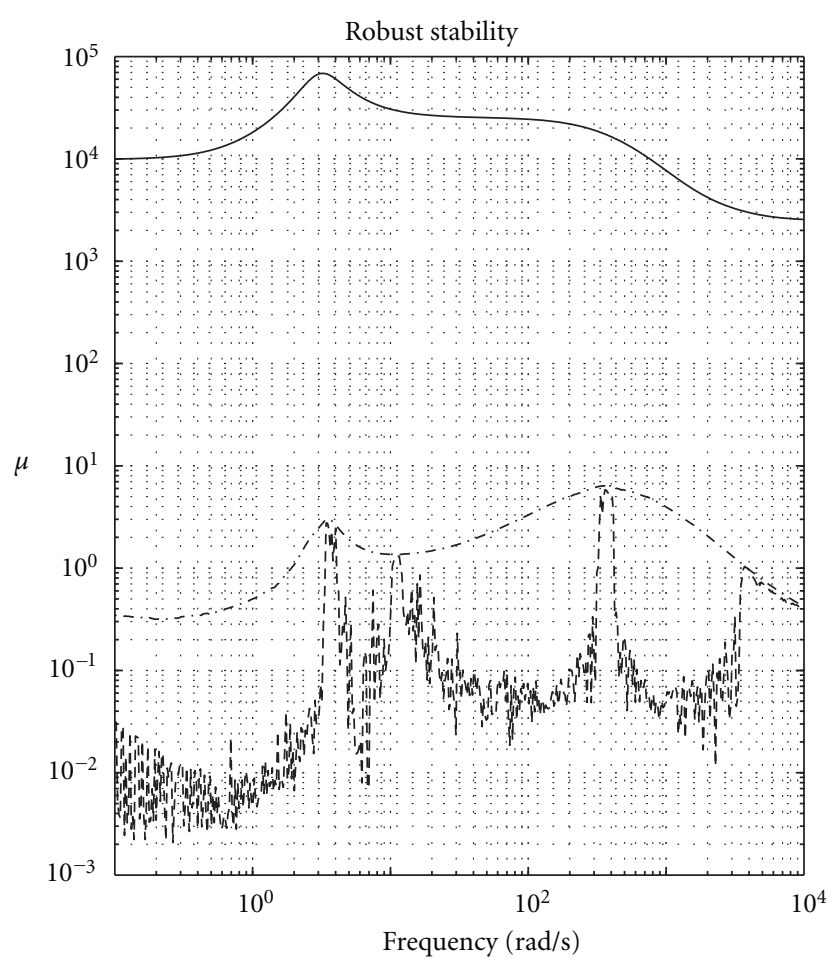

.-- Upper bound
--- Lower bound
$-\mu$ bound

(a)

Nominal performance (solid) and robust performance (dashed)

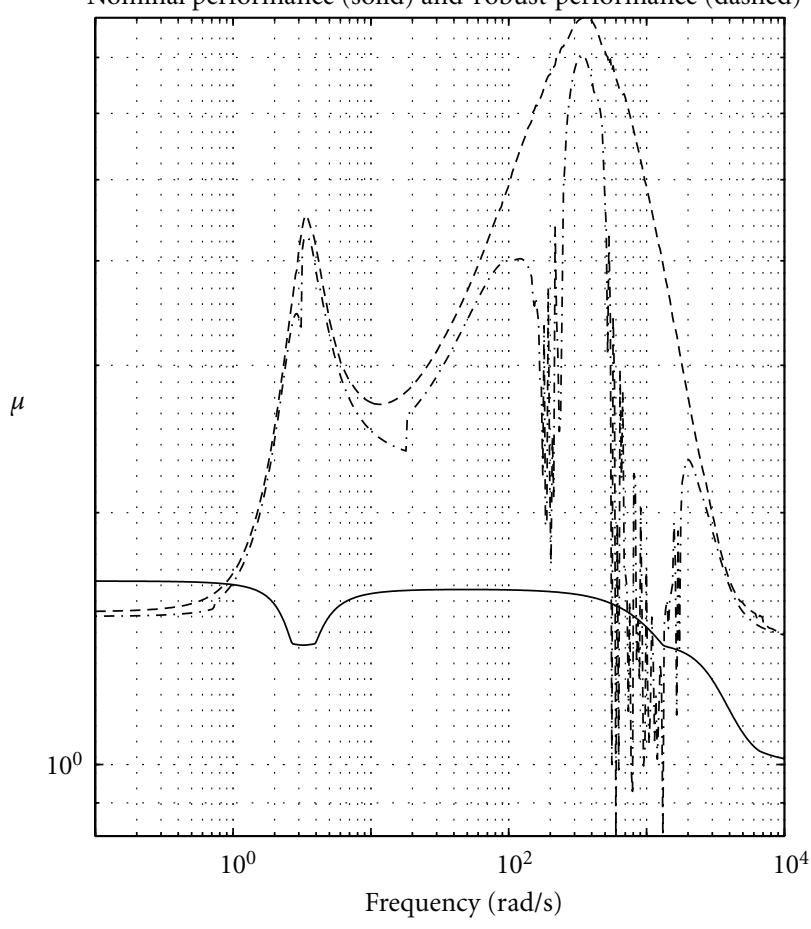

- Nom. perf.

- - - Upper bound

- - - Lower bound

(b)

FIGURE 8: Robust stability, nominal and robust performance function described by $\mu$-value with $\mu$-Synthesis. where:

$$
\begin{gathered}
\mathbf{K}_{S}=\left[\begin{array}{cccc}
k_{x A} & 0 & 0 & 0 \\
0 & k_{y A} & 0 & 0 \\
0 & 0 & k_{x B} & 0 \\
0 & 0 & 0 & k_{y B}
\end{array}\right], \\
\mathbf{K}_{I}=\left[\begin{array}{cccc}
k_{i x A} & 0 & 0 & 0 \\
0 & k_{i y A} & 0 & 0 \\
0 & 0 & k_{i x B} & 0 \\
0 & 0 & 0 & k_{i y B}
\end{array}\right], \\
\mathbf{i}_{c}(t)=\left[\begin{array}{l}
i_{c x A}(t) \\
i_{c y A}(t) \\
i_{c x B}(t) \\
i_{c y B}(t)
\end{array}\right],
\end{gathered}
$$

where the elements of $\mathbf{K}_{S}$ and $\mathbf{K}_{I}$, respectively, displacement and current gains matrices are

$$
\begin{aligned}
& k_{(x, y)(A, B)} \\
& =\left.\frac{\partial f\left(i_{c c(i x, i y)(A, B)}(t), q_{b(x, y)(A, B)}(t)\right)}{\partial q_{b(x, y)(A, B)}}\right|_{\substack{i_{c(i x, i y)(A, B)}=0 \\
q b(x, y)(A, B)=0}} \\
& =\left.2 k \frac{\left(i_{0(A, B)}-i_{c(i x, i y)(A, B)}\right)^{2}\left(g_{0(A, B)}+q_{b(x, y)(A, B)}\right)}{\left(g_{0(A, B)}+q_{b(x, y)(A, B)}\right)^{4}}\right|_{\substack{i_{c(i x, i y)(A, B)=0} \\
q_{b(x, y)(A, B)=0}}} \\
& =2 k_{(A, B)} \frac{i_{0(A, B)}^{2}}{g_{0(A, B)}^{3}}, \\
& k_{(i x, i y)(A, B)} \\
& =\left.\frac{\partial f\left(i_{c(i x, i y)(A, B)}(t), q_{b(x, y)(A, B)}(t)\right)}{\partial i_{c(i x, i y)(A, B)}}\right|_{\begin{array}{l}
i_{c(i x, i y)(A, B)=0} \\
q_{b(x, y)(A, B)}=0
\end{array}} \\
& =\left.2 k \frac{\left(i_{0(A, B)}-i_{c(i x, i y)(A, B)}\right)}{\left(g_{0(A, B)}+q_{b(x, y)(A, B)}\right)^{2}}\right|_{\substack{i_{c(i x, i y)(A, B)}=0 \\
q_{b(x, y)(A, B)=0}}} \\
& =2 k_{(A, B)} \frac{i_{0(A, B)}}{g_{0(A, B)}^{2}},
\end{aligned}
$$

where

$$
k_{(A, B)}=\frac{1}{4} \mu_{0} N_{(A, B)}^{2} A_{g(A, B)} \cos \beta
$$

is the multiplicative constant carried out from principle of virtual work and $\beta$ is the angle of two poles of one electromagnet. 


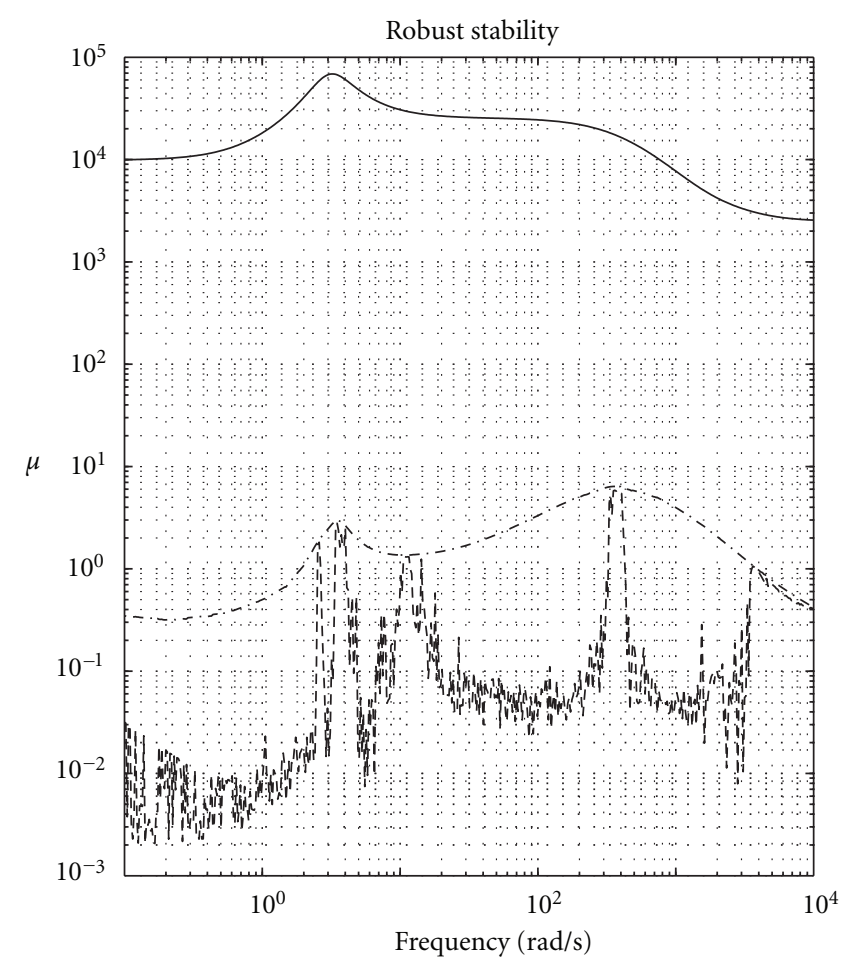

-.- - Upper bound

- - - Lower bound

- $\operatorname{Sub}\left(H_{\infty}\right)$ bound

(a)

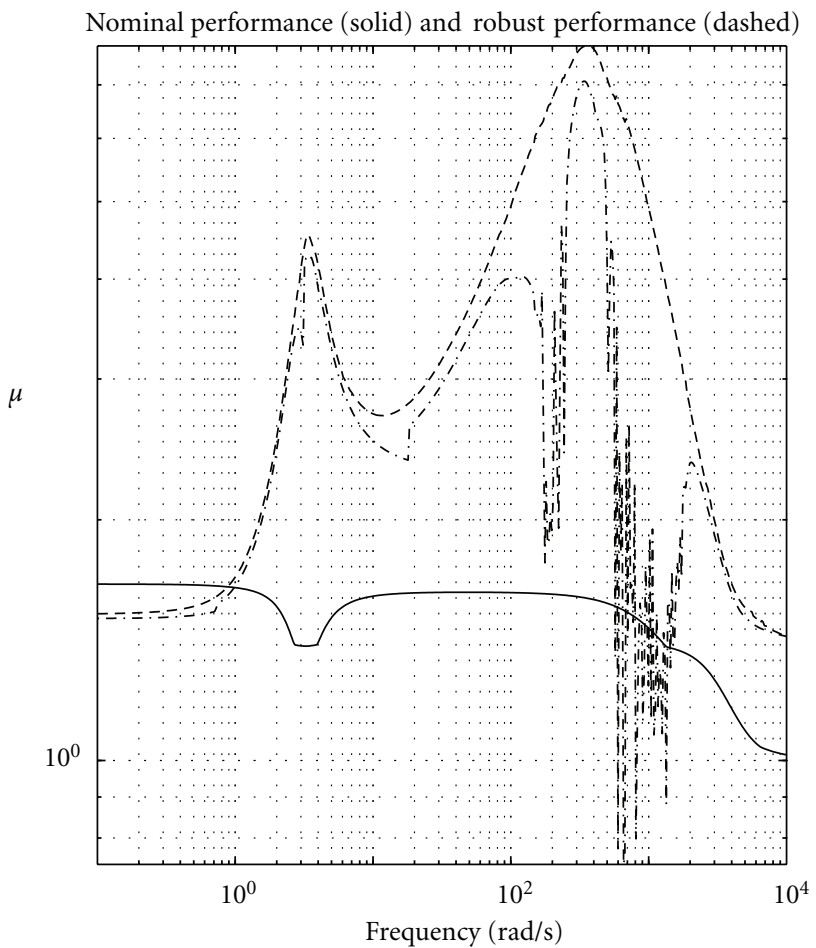

- Nom. perf.

- - - Upper bound

...- Lower bound

(b)

FIGURE 9: Robust stability, nominal and robust performance function described by $\mu$-value with $\operatorname{Sub}\left(H_{\infty}\right)$.

In this paper, the value of displacement and current gains are taken from a real model of radial active magnetic bearing produced by SKF. The physical meaning on these gain matrices is related to the reaction force produced for the unit of displacement and current, respectively, for displacement and current gains

Due to the transformation coordinates (5), we transform (9a) $-(9 d)$ in

$$
\mathbf{f}\left(\mathbf{i}_{c}(t), \mathbf{q}_{b}(t)\right) \approx \mathbf{K}_{S} \mathbf{B}_{\Theta \operatorname{disp}} \mathbf{B}_{\mathrm{mag}}^{\mathrm{T}} \mathbf{q}_{g}(t)+\mathbf{K}_{I} \mathbf{i}_{c}(t)
$$

But we do not have to forget that mathematical model in a state space form considers the matrix product $\mathbf{B}_{\Theta \mathrm{mag}} \mathbf{B}_{\mathrm{mag}}$ before $\mathbf{f}\left(\mathbf{i}_{c}(t), \mathbf{q}_{b}(t)\right)$ as in (6).

\section{Controller}

In order to provide a stabilizing effect to control the position of the rotor, a suitable control system must be performed because no magnetic levitation can be stabilized without controller $[1,4]$. Here we performed three different controllers or rather loop-shaping design, $\mu$-synthesis, and
$\operatorname{sub}\left(H_{\infty}\right)$ robust control according to the mathematical model (11) with the assumption

$$
\begin{aligned}
{\left[\begin{array}{c}
\dot{\mathbf{x}}_{1}(t) \\
\dot{\mathbf{x}}_{2}(t)
\end{array}\right]=} & {\left[\begin{array}{cc}
\mathbf{0} & \mathbf{I}^{4 \times 4} \\
\left(\overline{\mathbf{M}}^{-1} \mathbf{K}_{S}\right)^{4 \times 4} & -\overline{\mathbf{M}}^{-1}(\Omega \overline{\mathbf{G}})
\end{array}\right]\left[\begin{array}{l}
\mathbf{x}_{1}(t) \\
\mathbf{x}_{2}(t)
\end{array}\right] } \\
& +\left[\begin{array}{cc}
\mathbf{0} & \mathbf{0}^{4 \times 4} \\
-\mathbf{P}_{M}^{4 \times 4} & -\Omega \overline{\mathbf{M}}^{-1} \mathbf{P}_{G}
\end{array}\right]\left[\begin{array}{l}
\mathbf{u}_{M} \\
\mathbf{u}_{G}
\end{array}\right]+\left[\begin{array}{c}
\mathbf{0}^{4 \times 4} \\
\overline{\mathbf{M}}^{-1} \mathbf{K}_{I}
\end{array}\right] \mathbf{u}(t),
\end{aligned}
$$$$
\left[\begin{array}{l}
\mathbf{z}_{1}(t) \\
\mathbf{z}_{2}(t)
\end{array}\right]=\left[\begin{array}{cc}
\left(\overline{\mathbf{M}}^{-1} \mathbf{K}_{S}\right)^{4 \times 4} & -\overline{\mathbf{M}}^{-1}(\Omega \overline{\mathbf{G}}) \\
\mathbf{0} & (\Omega \overline{\mathbf{G}})^{4 \times 4}
\end{array}\right]\left[\begin{array}{l}
\mathbf{x}_{1}(t) \\
\mathbf{x}_{2}(t)
\end{array}\right]
$$

$$
\begin{gathered}
+\left[\begin{array}{cc}
-\mathbf{P}_{M}^{4 \times 4} & -\Omega \overline{\mathbf{M}}^{-1} \mathbf{P}_{G} \\
\mathbf{0} & \mathbf{0}^{4 \times 4}
\end{array}\right]\left[\begin{array}{l}
\mathbf{u}_{M} \\
\mathbf{u}_{G}
\end{array}\right]+\left[\begin{array}{c}
\overline{\mathbf{M}}^{-1} \mathbf{K}_{I} \\
\mathbf{0}^{4 \times 4}
\end{array}\right] \mathbf{u}(t), \\
\mathbf{y}(t)_{\text {sensor }}=\left[\left(\mathbf{B}_{\Theta \text { disp }} \mathbf{B}_{\text {sensor }}\right)^{4 \times 4} \mathbf{0}^{4 \times 4}\right]\left[\begin{array}{ll}
\mathbf{x}_{1}^{\mathrm{T}}(t) & \mathbf{x}_{2}^{\mathrm{T}}(t)
\end{array}\right]^{\mathrm{T}},
\end{gathered}
$$




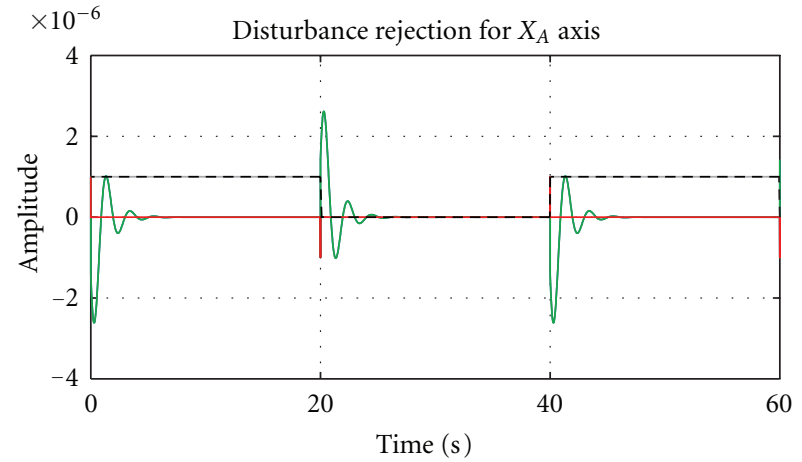

(a)

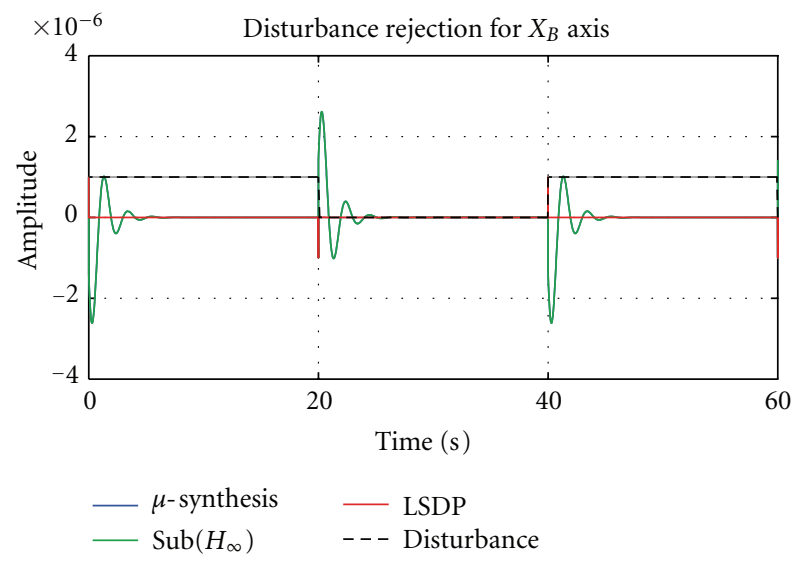

(c)

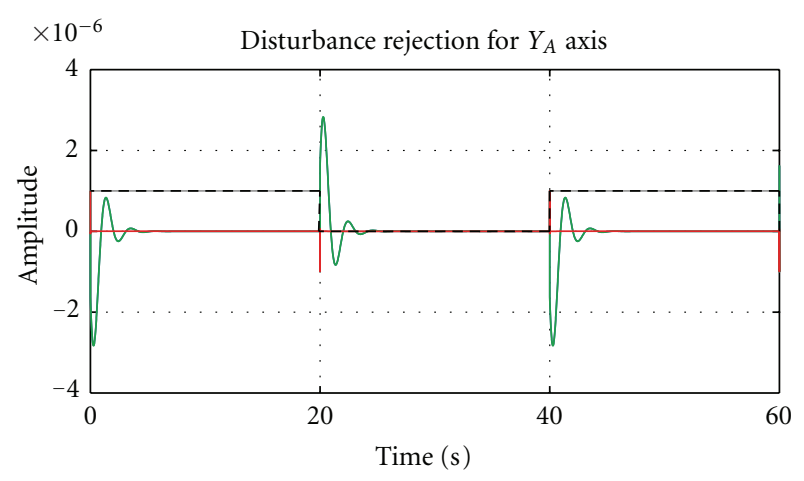

(b)

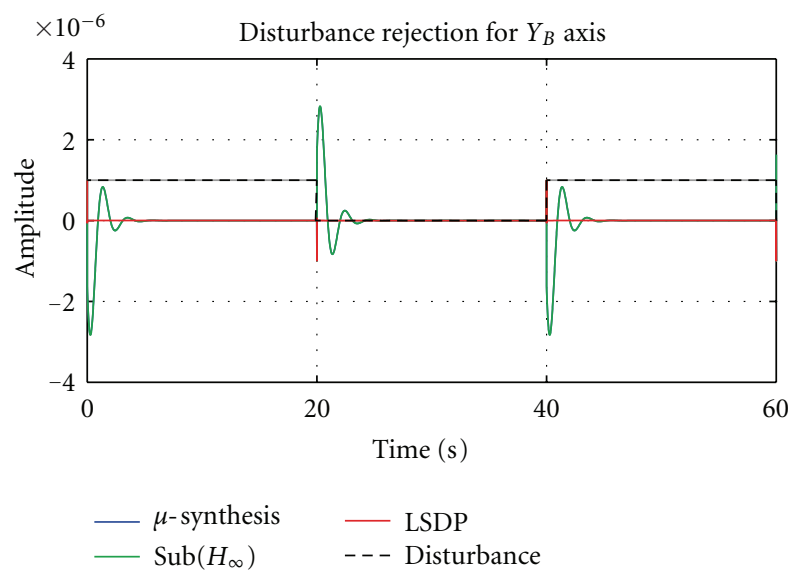

(d)

FIGURE 10: Comparison via simulation for disturbance rejection test.

$$
\begin{aligned}
& \mathbf{x}_{1}(t)=\left[\begin{array}{llll}
x_{g}(t) & \gamma(t) & y_{g}(t) & \vartheta(t)
\end{array}\right]^{\mathrm{T}}, \\
& \mathbf{x}_{2}(t)=\left[\begin{array}{llll}
\dot{x}_{g}(t) & \dot{\gamma}(t) & \dot{y}_{g}(t) & \dot{\vartheta}(t)
\end{array}\right]^{\mathrm{T}}, \\
& \mathbf{u}(t)=\mathbf{i}_{c}(t),
\end{aligned}
$$$$
\left[\begin{array}{c}
\mathbf{u}_{M} \\
\mathbf{u}_{G}
\end{array}\right]=\left[\begin{array}{cc}
\Delta_{M} & \mathbf{0}^{4 \times 4} \\
\mathbf{0}^{4 \times 4} & \Delta_{G}
\end{array}\right]\left[\begin{array}{l}
\mathbf{z}_{1}(t) \\
\mathbf{z}_{2}(t)
\end{array}\right] .
$$

For all kind of robust control systems performed in this paper, a state space equation in a package form is adopted for this purpose. The package form characterized by the introduction of all inputs in terms of uncertainties, disturbances, and control signal is introduced into plant as in

$$
\mathbf{G}=\left[\begin{array}{ccc}
\mathbf{A} & \mathbf{B}_{1} & \mathbf{B}_{2} \\
\mathbf{C}_{1} & \mathbf{D}_{11} & \mathbf{D}_{12} \\
\mathbf{C}_{2} & \mathbf{D}_{21} & \mathbf{D}_{22}
\end{array}\right],
$$

where

$$
\begin{aligned}
& \mathbf{A}=\left[\begin{array}{cc}
\mathbf{0} & \mathbf{I}^{4 \times 4} \\
\left(\overline{\mathbf{M}}^{-1} \mathbf{K}_{S}\right)^{4 \times 4} & -\overline{\mathbf{M}}^{-1}(\Omega \overline{\mathbf{G}})
\end{array}\right], \\
& \mathbf{B}_{1}=\left[\begin{array}{cc}
\mathbf{0} & \mathbf{0}^{4 \times 4} \\
-\mathbf{P}_{M}^{4 \times 4} & -\overline{\mathbf{M}}^{-1} \mathbf{P}_{G}
\end{array}\right], \quad \mathbf{B}_{2}=\left[\begin{array}{c}
\mathbf{0}^{4 \times 4} \\
\overline{\mathbf{M}}^{-1} \mathbf{K}_{I}
\end{array}\right], \\
& \mathbf{C}_{1}=\left[\begin{array}{cc}
\left(\overline{\mathbf{M}}^{-1} \mathbf{K}_{S}\right)^{4 \times 4} & -\overline{\mathbf{M}}^{-1}(\Omega \overline{\mathbf{G}}) \\
\mathbf{0} & (\Omega \overline{\mathbf{G}})^{4 \times 4}
\end{array}\right], \\
& \mathbf{D}_{11}=\left[\begin{array}{cc}
-\mathbf{P}_{M}^{4 \times 4} & -\overline{\mathbf{M}}^{-1} \mathbf{P}_{G} \\
\mathbf{0} & \mathbf{0}^{4 \times 4}
\end{array}\right], \quad \mathbf{D}_{12}=\left[\begin{array}{c}
\overline{\mathbf{M}}^{-1} \mathbf{K}_{I} \\
\mathbf{0}^{4 \times 4}
\end{array}\right],
\end{aligned}
$$

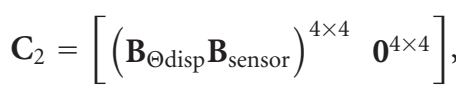

$$
\begin{aligned}
& \mathbf{D}_{21}=\mathbf{0}^{4 \times 8}, \quad \mathbf{D}_{22}=\mathbf{0}^{4 \times 8} \text {. }
\end{aligned}
$$

Usually a rotor supported by active magnetic bearing needs to reach some desired performances that are described by weighting functions. The weighting functions introduced 


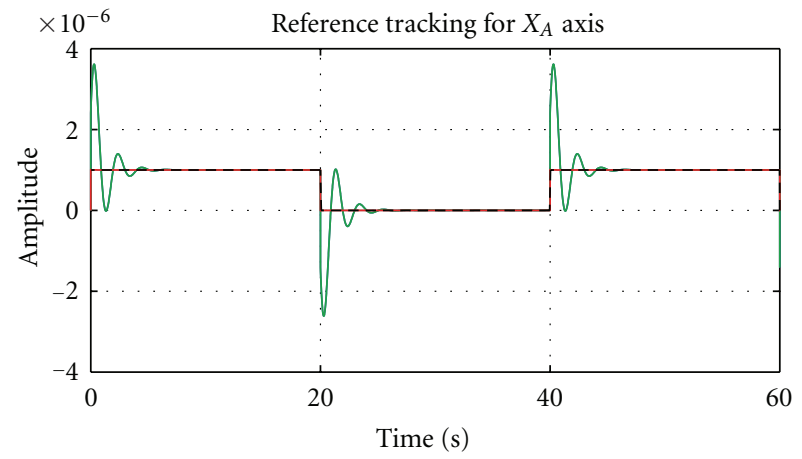

(a)

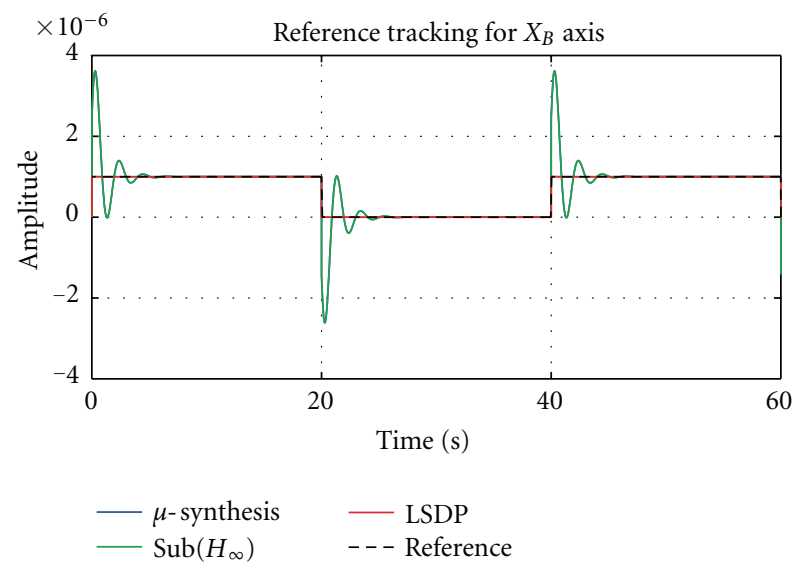

(c)

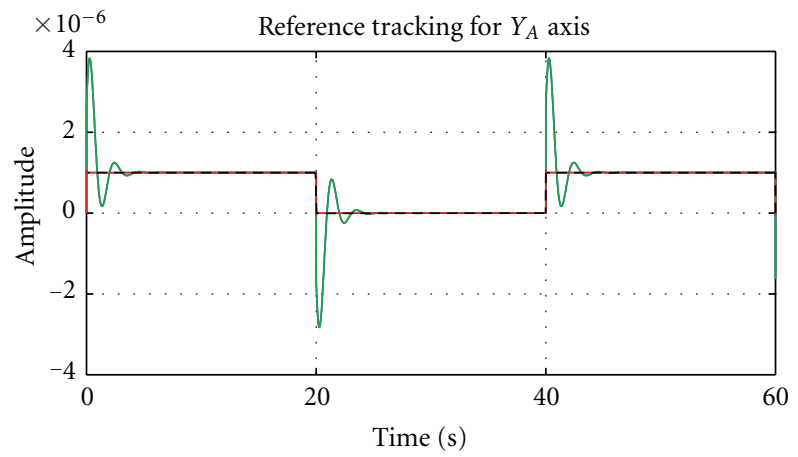

(b)

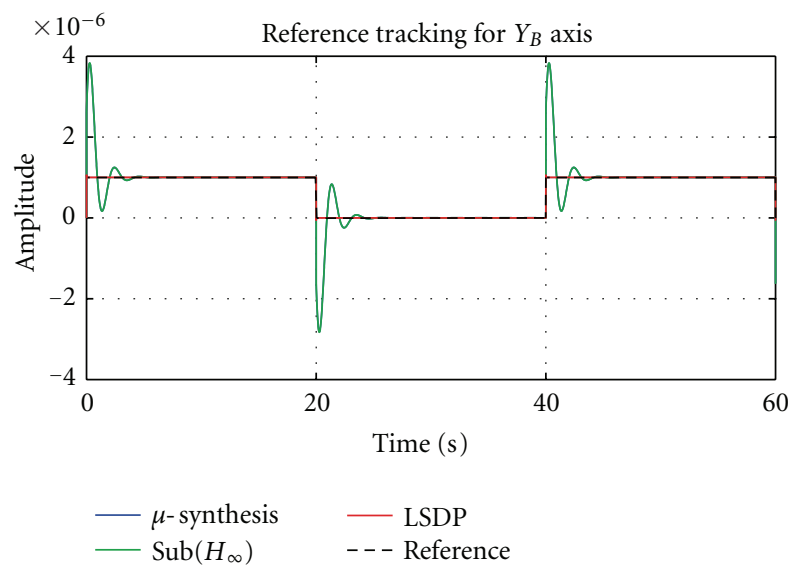

(d)

FIGURE 11: Comparison via simulation for reference tracking test.

in the plant are relative to position and control signal performances in order to impose limits in the current value and maximum displacement of each rotor's section. The weighting functions are transfer functions in Laplace domain describing the desired dynamic behavior of the rotor's section varying by the excitation frequency. The expressions (15) and (16) are the weighting functions for position and control input, respectively,

$$
\begin{gathered}
W_{p}=\frac{s^{2}+1.8 s+10}{s^{2}+8 s+0.01}, \\
W_{u}=10^{-5} .
\end{gathered}
$$

The weighting function described in (15) is applied to the displacements of the rotor's sections, while the control signal is scaled according to (16). The transfer functions are taken by considering that the system has to be subjected to an attenuation of disturbances. This attenuation can be developed by maintaining the frequency response of the shaped plant by the controller well below the frequency response of (15) in the frequency range of interest. The expression of (15) can be obtained by MATLab commands by specifying the upper and lower limits of range of frequency. The block scheme is shown in the Figure 2, where $G_{\mathrm{mds}}$ is the nominal plant meant without uncertainties, $K$ is the controller, $d$ the disturbances, and $e_{p}$ and $e_{u}$ the outputs of weighting function with regard to the position and control signal, respectively.

The presence of weighting functions produces an increase of state vector's variables so that the new plant is $\mathrm{P}$ as shown in Figure 3.

All controllers used in this paper are characterized by a common concept or rather the robustness. The robustness is meant in a double way: robust stability and robust performance. The closed-loop system achieves robust stability if it is internally stable for all possible plant models $G=$ $F\left(G_{\mathrm{mds}}, \Delta\right)$. In the present case, this means that the system must remain stable for any value of $\delta_{m, I_{P}, I_{P}}$.

Since those weighting functions are introduced in order to provide some characteristic on the system's output, the robust performance criterion (17) is introduced for all $G=$ $F\left(G_{\mathrm{mds}}, \Delta\right)$

$$
\begin{gathered}
\left\|\left[\begin{array}{c}
W_{p}(I+G K)^{-1} \\
W_{u} K(I+G K)^{-1}
\end{array}\right]\right\|_{\infty}<1, \\
{\left[\begin{array}{c}
\mathbf{e}_{p} \\
\mathbf{e}_{u}
\end{array}\right]=\left[\begin{array}{c}
\mathbf{W}_{p}(\mathbf{I}+\mathbf{G K})^{-1} \\
\mathbf{W}_{u} \mathbf{K}(\mathbf{I}+\mathbf{G K})^{-1}
\end{array}\right] \mathbf{d},}
\end{gathered}
$$

where $\Delta$ can be structured or unstructured uncertainty matrix; in any case it must satisfy the norm condition or 
TABLE 1: Data for simulation.

\begin{tabular}{llc}
\hline Symbol & Description & S.I. \\
\hline $\bar{m}$ & mass of rotor & $2.3 \mathrm{Kg}$ \\
$\bar{I}_{P}$ & polar moment of inertia & $8 \times 10^{-4} \mathrm{Kg} \cdot \mathrm{m}^{2}$ \\
$\bar{I}_{T}$ & transverse moment of inertia & $6 \times 10^{-2} \mathrm{Kg} \cdot \mathrm{m}^{2}$ \\
$l_{b A}$ & distance bearing $A$ from centre of & $0.241 \mathrm{~m}$ \\
& mass $G$ & \\
$l_{b B}$ & distance bearing $B$ from centre of & $0.139 \mathrm{~m}$ \\
& mass $G$ & \\
$l_{s A}$ & distance sensor $A$ from centre of & $0.241 \mathrm{~m}$ \\
& mass $G$ & \\
$l_{s B}$ & distance sensor $B$ from centre of & $0.119 \mathrm{~m}$ \\
$A_{g(A, B)}$ & mass $G$ & pole surface \\
$g_{0(A, B)}$ & nominal gap & $6.6 \times 10^{-5} \mathrm{~m}{ }^{2}$ \\
$\alpha$ & slope of bearings axis & $3.81 \times 10^{-4} \mathrm{~m}$ \\
$\beta$ & angle of two electromagnet poles & $45^{\circ}$ \\
$\alpha$ & slope of bearings axis & $45^{\circ}$ \\
$P_{m, I_{T}, I_{P}}$ & uncertainties percentage & $45^{\circ}$ \\
$\delta_{m, I_{T}, I_{P}}$ & range of uncertainties & $10 \%$ \\
$k_{(x, y)(A, B)}$ & displacement gain & {$[-1,1]$} \\
$k_{(i x, i y)(A, B)}$ & current gain & $144000 \mathrm{~N} / \mathrm{m}$ \\
\hline & & $38 \mathrm{~N} / \mathrm{A}$ \\
\hline
\end{tabular}

rather: $\|\Delta\|_{\infty}<1$ for robust performances and where vector d in (18) is the disturbances on the system.

Hence, the performance criterion is that the transfer functions from $\mathbf{d}$ to $\mathbf{e}_{p}$ and $\mathbf{e}_{u}$ should be small in the sense of \|\|$_{\infty}$ for all possible uncertain transfer matrices $\Delta$.

\section{Results, Simulations, and Discussion}

The simulations are performed by considering the data contained in Table 1.

Another set of data are referred to the transfer function introduced in the plant of our system. These transfer functions are essential if a certain performance must be obtained; these performances are usually referred in the frequency domain for example. Some authors usually introduce scalar weighting function in order to describe a certain constant value they want to obtain as a particular output. In this paper, we introduce a transfer function in the output signals or rather the displacement. The introduction of transfer function in Laplace domain " $\mathrm{s}$ " means that the displacement must be characterized by a certain dynamic behavior according the frequency variable. This technique is commonly used above all when a flexible structure is taken into account or when some nodes are subjected to vibrations such as in this case. Figures 4, 5, and 6 show the frequency response of weighting function relative to the displacement performances in order to analyze the sensitivity function. This is made for all controlled axis of each radial active magnetic bearing. Figure 4 shows the sensitivity function for the loop-shaping controller design, and we can see that the system has a good attenuation of disturbances until a certain value of frequency equals $10^{3} \mathrm{~Hz}$ for Loop-Shaping Design Procedure (LSPD) controller.

Figures 5 and 6 show that $\mu$-synthesis and $\operatorname{Sub}\left(H_{\infty}\right)$ robust control are not able to maintain a good rejection of disturbances for the entire range of frequency required for the system.

In a physical sense, the $\mu$ value represents an amplification coefficient of the effect produced by an exogenous excitation on the system. In a mathematical sense, it is determined by the following expression:

$$
\mu\left(\Delta, \mathbf{G}_{z w}\right)=\frac{1}{\min \left(\bar{\sigma}(\Delta): \operatorname{det}\left(\mathbf{I}-\mathbf{G}_{z w} \boldsymbol{\Delta}\right)\right)},
$$

where $\bar{\sigma}(\Delta)$ is the singular value of relative input-output plant as a function of $\Delta$.

Figure 7 discusses the robust stability condition for loopshaping design procedure and shows that system has robust stability until a value of frequency of $10^{3} \mathrm{~Hz}$. After this value of frequency, the system has a pick outside the unitary value and goes back under one when the frequency approaches to the value of $10^{4} \mathrm{~Hz}$ (upper and lower bound). The loopshaping design shows good performances until $10^{3} \mathrm{~Hz}$ for upper and lower bound since $\mu<1$. The loop-shaping design shows also a good rejection of disturbances as shown in the sensitivity function in Figure 4; in fact it has maintained itself well below the transfer function (15).

Figure 8 shows that robust stability for the $\mu$-synthesis is not maintained in the range of frequency near $\left[10^{0}, 10^{3}\right] \mathrm{Hz}$ so that the system does not have robust stability in that range. Figure 8 shows that, in the study of robust performances, the $\mu$ value is more than one for all range of operating frequency. This means the state of uncertainties introduced in this work induces the system to have bad robust performances also for the nominal plant. The same behavior is obtained by the $\operatorname{Sub}\left(H_{\infty}\right)$ control.

Obviously the previous results affect the dynamic behavior of the entire system. According to Figure 3, the study of reference's tracking and disturbances rejection is performed. It is built for all studied controllers. In Figure 10, the disturbance rejection test is performed according to a simulation characterized by a range of time of sixty seconds and a disturbance injection built as a square wave (black line) with a period of $20 \mathrm{~s}$ and amplitude of $10^{-6} \mathrm{~m}$. All three controllers implemented are capable to support the requirements to reject the disturbance in a different way. The controllers $\operatorname{Sub}\left(H_{\infty}\right)$ and $\mu$-Synthesis are characterized by the same pattern and the same period of oscillation, and they are perfectly superimposed. Their dynamic behavior is typical of damped system where a certain overshoot's value is present and different from the previous controllers. The loopshaping controller (red line) provides a good performance for the disturbance rejection due to the short period to extinct the transient response and small overshoot's value if compared with those offered by the previous controllers; it has a good disturbance rejection and good performance because the it has a $\mu$ value lower than one. In all cases, the controllers are able to reject the disturbances by leading all suspended section to maintain the operating point position. Figure 11 shows the reference's tracking simulations. 
The controllers are characterized by state space representation (20) with four input positions and four control current outputs:

$$
\begin{gathered}
\dot{\mathbf{x}}(t)=\mathbf{A} \mathbf{x}(t)+\mathbf{B u}(t), \\
\mathbf{y}(t)=\mathbf{C} \mathbf{x}(t), \\
\mathbf{u}(t)=\mathbf{q}(t)_{\text {sensor }}, \\
\mathbf{y}(t)=\mathbf{i}_{c}(t)
\end{gathered}
$$

\section{Conclusions}

This paper shows that a comparison of three different control systems is built for a suspended rotor by active magnetic bearings. The comparison shows that loop-shaping design procedure provides the best performance to eliminate the disturbances and to follow the reference's tracking when a certain required performances on position and control current are necessary. The present study shows that, once the weighting functions are introduced, only the loop-shaping design procedure is able to lead the system with robust stability and robust performance. The next development is to produce the previous discussion for the flexible rotor under the assumption that sensors are not colocated and with a state of uncertainties on displacement and current control gains.

\section{References}

[1] G. Schweitzer, H. Bleuler, and A. Traxler, Active Magnetic Bearings, Vdf Hochschulverlag AG, Zurich, Switzerland, 1994.

[2] G. Genta, Dynamics of Rotating Systems, Mechanical Engineering Series, Springer, Torino, Italy, 2005.

[3] G. Barbaraci and G. Virzì Mariotti, "Controllo subottimo per un albero rotante in levitazione magnetica attiva," in Proceedings of the 38th Convegno Nazionale AIAS, Torino, Italy, Settembre 2009.

[4] G. Schweitzer and E. H. Maslen, Magnetic Bearings: Theory, Design and Application to Rotating Machinery, Springer, Zurich, Switzerland, 2009.

[5] G. Barbaraci, A. H. Pesch, and J. T. Sawicki, "Experimental investigations of minimum power consumption optimal control for variable speed amb rotor," in Proceedings of the ASME 2010 International Mechanical Engineering Congress \& Exposition (IMECE '10), pp. 1-10, Vancouver, Canada.

[6] D. Bugajski, D. Enns, and A. Tannenbaum, "Synthesis methods for robust nonlinear control," in Proceedings of the American Control Conference, pp. 531-535, June 1993.

[7] C. R. Knospe, R. L. Fittro, and L. S. Stephens, "Control of a high speed machining spindle via $\mu$-synthesis," in Proceedings of the 1997 IEEE International Conference on Control Applications, pp. 912-917, Hartford, Conn, USA, October 1997.

[8] J. D. Wu and J. H. Lin, "Implementation of an active vibration controller for gear-set shaft using $\mu$-analysis," Journal of Sound and Vibration, vol. 281, no. 3-5, pp. 1037-1055, 2005.

[9] S. Kaitwanidvilai and M. Parnichkun, "Design of structured controller satisfying $H_{\infty}$ loop shaping using evolutionary optimization: application to a pneumatic robot arm," Engineering Letters, vol. 16, no. 2, 2003. 

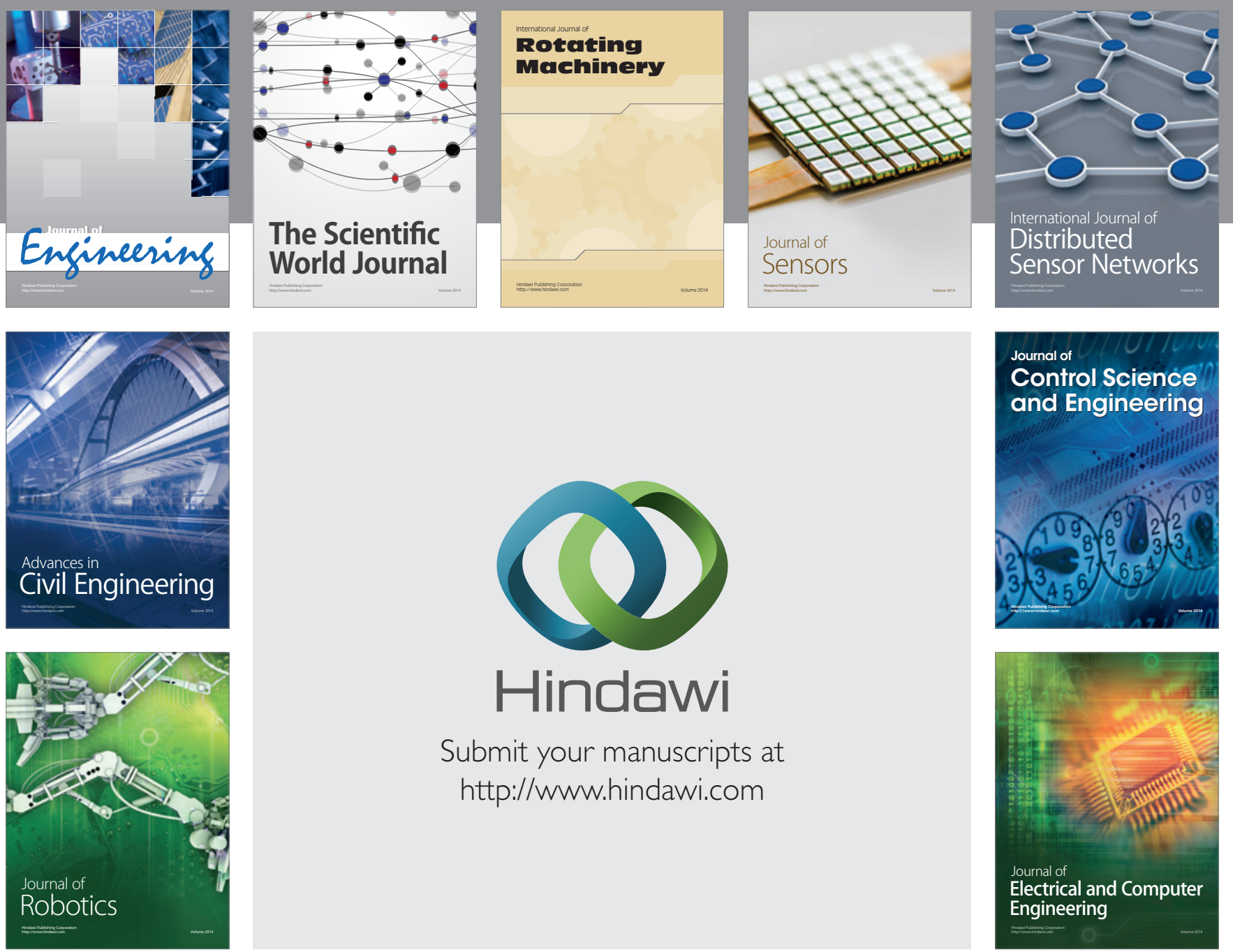

Submit your manuscripts at

http://www.hindawi.com
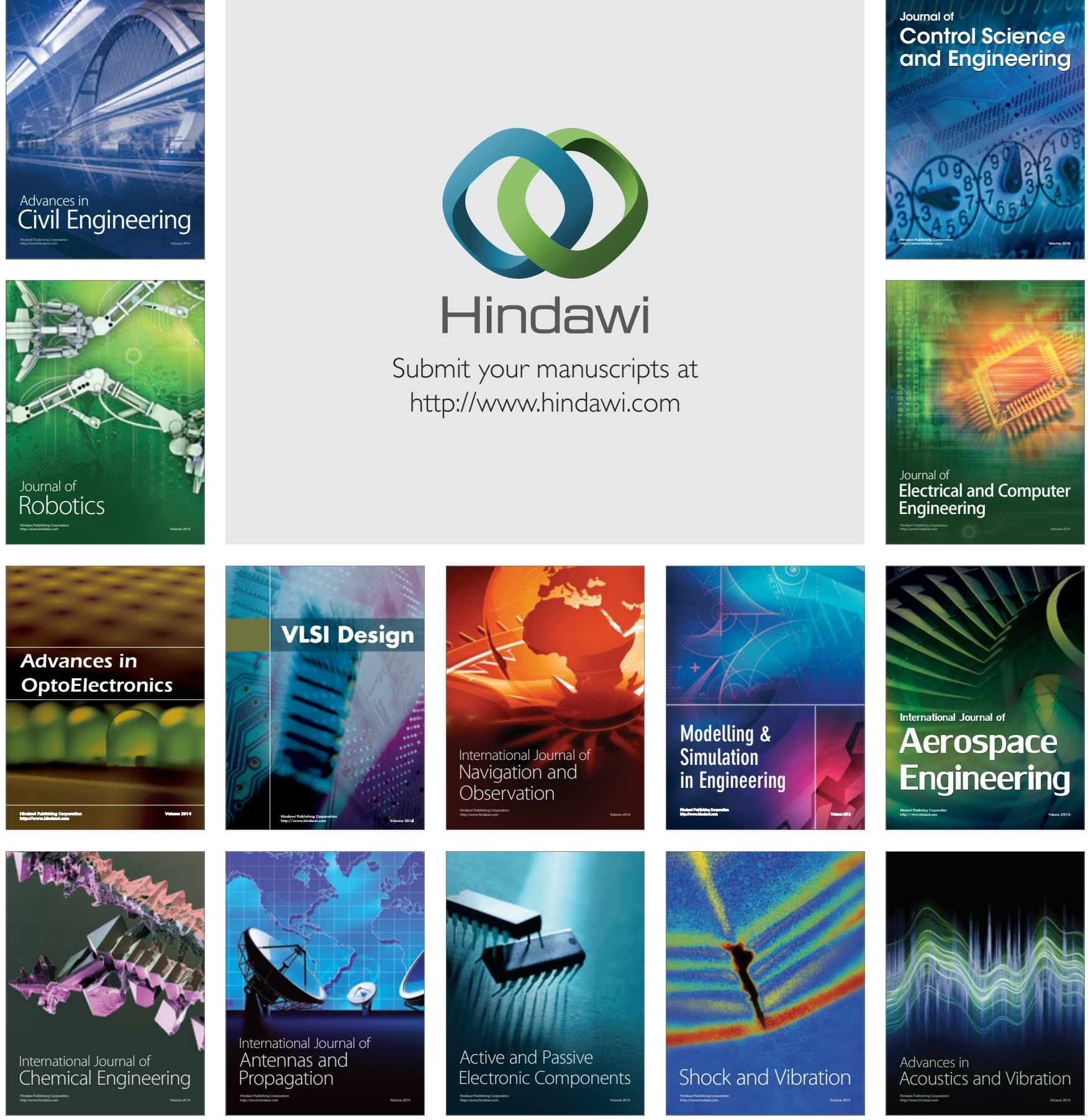